\title{
A phenomenological and extended continuum approach for modelling non-equilibrium flows
}

Received: 26 February 2007 / Accepted: 28 June 2007 / Published online: 31 July 2007

(C) Springer-Verlag 2007

\begin{abstract}
This paper presents a new technique that combines Grad's 13-moment equations (G13) with a phenomenological approach to rarefied gas flows. This combination and the proposed solution technique capture some important non-equilibrium phenomena that appear in the early continuum-transition flow regime. In contrast to the fully coupled 13-moment equation set, a significant advantage of the present solution technique is that it does not require extra boundary conditions explicitly; Grad's equations for viscous stress and heat flux are used as constitutive relations for the conservation equations instead of being solved as equations of transport. The relative computational cost of this novel technique is low in comparison to other methods, such as fully coupled solutions involving many moments or discrete methods. In this study, the proposed numerical procedure is tested on a planar Couette flow case, and the results are compared to predictions obtained from the direct simulation Monte Carlo method. This test case highlights the presence of normal viscous stresses and tangential heat fluxes that arise from non-equilibrium phenomena, which cannot be captured by the Navier-Stokes-Fourier constitutive equations or phenomenological modifications.
\end{abstract}

Keywords Flows in micro-electromechanical systems (MEMS) and nano-electromechanical systems (NEMS) · Micro- and nano- scale flow phenomena - Rarefied gas dynamics · Non-continuum effects · Non-equilibrium gas dynamics

PACS 47.61.Fg, 47.61.-k, 47.45.-n, 47.61.Cb, 47.70.Nd

\section{Introduction}

Micro-electro-mechanical systems (MEMS) have found many applications in industrial and process systems, biomedical devices, environmental control devices, micro-processor cooling, and high precision printing. Micro-ducts, micro-heat-exchangers, micro-pumps, and micro-air-vehicles are now commonly used terms. Rapid progress in micro-engineering has not been matched by an increased understanding of the fundamental physics occurring in such small-scale domains. One particularly active research area of emerging importance

Communicated by M. Slemrod

S. Mizzi $(\varangle) \cdot$ R. W. Barber · D. R. Emerson

Centre for Microfluidics and Microsystems Modelling, Computational Science and Engineering Department,

CCLRC Daresbury Laboratory, Keckwick Lane, Daresbury, WA4 4AD Warrington, UK

E-mail: s.mizzi@dl.ac.uk

J. M. Reese

Mechanical Engineering Department, University of Strathclyde, James Weir Building, G1 1XJ Glasgow, UK

S. K. Stefanov

Institute of Mechanics, Bulgarian Academy of Sciences, Block 4, Acad. G. Bonchev Street, 1113 Sofia, Bulgaria 
is understanding the gas dynamics occurring in these miniaturized domains. This is because the general characteristics observed in macro-scale flows are not always applicable to micro-sized domains. For example, the Navier-Stokes-Fourier (NSF) fluid dynamic equations with conventional no-slip boundary conditions are no longer valid when the system characteristic length scale approaches the molecular mean free path of the gas [5].

The inadequacy of the NSF equations to represent gas dynamics in micro-domains stems from the fact that they are only able to describe flows which are close to equilibrium. Molecular collisions are the mechanism by which gas molecules equilibrate energy and momentum. Hence, if a gas is too rarefied, or is confined to micro-geometries, the number of collisions is reduced considerably relative to the bulk flow in the system, inducing non-equilibrium phenomena. If $\lambda$ is the mean free path, i.e., the average distance travelled by a gas molecule between successive collisions, and $H$ is the system characteristic length, then $K n=\lambda / H$, is a dimensionless parameter called the Knudsen number that gauges the degree of non-equilibrium.

The NSF equations can be obtained from the Boltzmann equation,

$$
\frac{\partial f}{\partial t}+c_{i} \frac{\partial f}{\partial x_{i}}+a_{i} \frac{\partial f}{\partial c_{i}}=\left.\frac{\partial f}{\partial t}\right|_{C},
$$

through approximations of $f$, the particle distribution function (PDF). Equation(1) is considered to be the fundamental governing equation of any dilute gas characterized by binary collisions. However, its solution is a non-trivial task due to the dimensional complexity of the collisional operator on the right hand side of Eq. (1). Various methods have been used to obtain a simpler approximation: each method attempting to retain acceptable accuracy in order to capture the fundamental gas dynamics. There are two main approaches.

In discrete molecular methods, the fluid is modelled using a microscopic formalism, i.e., as a collection of moving molecules which interact through collisions or very close proximity potentials. Such modelling can be performed using either statistical ensemble averages, e.g., direct simulation Monte Carlo (DSMC) [2], or deterministic methods, e.g., molecular dynamics (MD) [17]. Although such methods achieve a realistic picture, their application has been restricted to simple flows due to their computationally intensive nature.

Continuum modelling is another approach. The fluid is assumed continuous and infinitely divisible so it is possible to define velocity, density, pressure, and other properties at any point in space and time. This modelling approach can be sub-divided further. Extended gas dynamic continuum models can be derived by either perturbation methods, commonly known as the Chapman-Enskog expansion [4], or moment methods $[6,9]$. The usual conservation equations with the NSF constitutive relations are derived in each case [18]. The other category of continuum models is the use of simple phenomenological extensions to the governing conservation equations in order to capture non-equilibrium effects. The application of velocity slip [15] and temperature jump [19] boundary conditions as well as constitutive model scaling in the form of wall function methods [13] have been used in continuum-based approaches.

It has previously been shown that Grad's 13-moment equations are unable to capture the non-linear stress/ strain relationship in the near-wall region [14]. In this paper we focus on continuum-based methods with a unique combination of moment and phenomenological extensions in order to achieve a better description of non-equilibrium effects that are typical of gaseous flows in micron-sized domains. Combining the two methods not only improves the description of non-equilibrium phenomena but also retains the relatively low computational cost—a desirable feature for modelling tools used in engineering design applications.

\section{Extended hydrodynamics: the moment method}

The governing fluid conservation laws for mass, momentum and energy contain moments in the form of stress, $\sigma_{i j}=p \delta_{i j}+\sigma_{<i j>}=p \delta_{i j}+\tau_{i j}$ and heat flux, $q_{i}$. The angular brackets $<>$ indicate the traceless part of a tensor. Two options exist to derive relationships for these higher-order moments; (i) derive their transport equations, or (ii) obtain a constitutive relationship in the form of a closure approximation in terms of the five lower-order moments, i.e., $\rho, v_{i}$ and $T$.

One form of closure approximation is by considering a Hermite polynomial expansion of the PDF [6]. The resulting closure approximation for the first five moments are termed the Euler equations with the resulting constitutive relations being $\tau_{i j}=0$ and $q_{j}=0$. When transport relations (governing equations) for the viscous stress, $\tau_{i j}$, and heat flux, $q_{i}$, are derived, the moment equations are then of the form:

$$
\frac{\partial \tau_{i j}}{\partial t}+\frac{\partial \tau_{i j} v_{k}}{\partial x_{k}}+\frac{4}{5} \frac{\partial q_{<i}}{\partial x_{j>}}+\frac{\partial \rho_{<i j k>}}{\partial x_{k}}+2 p \frac{\partial v_{<i}}{\partial x_{j>}}+2 \tau_{k<i} \frac{\partial v_{j>}}{\partial x_{k}}=-\frac{\tau_{i j}}{\tau_{\mathrm{c}}}
$$


and

$$
\begin{aligned}
& \frac{\partial q_{i}}{\partial t}+\frac{\partial q_{i} v_{k}}{\partial x_{k}}+\frac{1}{2} \frac{\partial \rho_{r r<i k>}}{\partial x_{k}}+\frac{1}{6} \frac{\partial \rho_{r r s s}}{\partial x_{i}}-\frac{5}{2} \frac{p}{\rho} \frac{\partial p}{\partial x_{i}}-\frac{5}{2} \frac{p}{\rho} \frac{\partial \tau_{i k}}{\partial x_{k}}-\frac{\tau_{i k}}{\rho} \frac{\partial p}{\partial x_{k}}-\frac{\tau_{i j}}{\rho} \frac{\partial \tau_{j k}}{\partial x_{k}} \\
& +\frac{7}{5} q_{k} \frac{\partial v_{i}}{\partial x_{k}}+\frac{2}{5} q_{k} \frac{\partial v_{k}}{\partial x_{i}}+\frac{2}{5} q_{i} \frac{\partial v_{k}}{\partial x_{k}}+\rho_{<i j k>} \frac{\partial v_{j}}{\partial x_{k}}=-\operatorname{Pr} \frac{q_{i}}{\tau_{\mathrm{c}}}
\end{aligned}
$$

where the collision frequency is defined as $\tau_{\mathrm{c}}=\mu / p$.

Higher-order moments $\rho_{<i j k>}, \rho_{r r<i k>}$ and $\rho_{\text {rrss }}$ appear in the viscous stress and heat flux transport equations, Eqs. (2) and (3). Again, there are two options to obtain relationships for these higher-order moments: either using a closure approximation, or formulating further transport equations recursively until a closure is defined. A closure for the first 13 moments yields the Grad 13-moment equations (G13), whereby $\rho_{<i j k>}=0$, $\rho_{r r<i k>}=7 R T \tau_{i k}$ and $\rho_{r r s s}=15 \mathrm{p}^{2} / \rho$. The NSF equations can be derived from both moment equation sets described here. Regularization of the Euler equations yields the NSF expressions [18].

A fully coupled solution of any moment equation set larger than five requires additional boundary conditions, which may be derived from kinetic schemes [18]. The derivation of such boundary conditions is a non-trivial task and becomes more complex as more moments are considered [7]. In addition, a coupled solution of systems with a large number of moments incurs a computational cost that is comparable to that of discrete methods, although the consideration of more moments should yield a better approximation to the Boltzmann equation.

\section{A method of differential iteration using moment equations}

The specific structure of the moment equations lends itself to being decoupled into two sub-systems and solved without the need for any additional boundary conditions. The Maxwellian iteration, best illustrated in [8], decouples the conserved variables, i.e., $\rho, v_{i}$ and $T$ from all the higher-order moments $\tau_{i j}, q_{j}, \rho_{<i j k>}, \rho_{r r<i k>}$, $\rho_{\text {rrss }}$, etc. The first Maxwellian iteration of the G13 moment equations yields the NSF equations. Liu proposed a decoupling method along these lines with the iterations being weighted [11] and the method has been shown to be mathematically consistent and convergent [12]. In order to illustrate this approach, the G13 moment set will be used as an example.

We denote the two sub-systems as I and II. System I is equivalent to the conservation laws together with constitutive relations for the viscous stress and the heat flux. System II consists of all the transport equations of the non-equilibrium variables which will be used as modified constitutive relations in system I, i.e., the viscous stresses, heat fluxes, and all higher-order moments for which the boundary conditions are unknown. It is assumed that the non-equilibrium fluid property fields are continuous up to the boundaries. Such an assumption is plausible even though discontinuities might exist at the wall boundaries in a similar way to velocity slip and temperature jump. The value on the gas side is the value that ultimately is of significance to the solution and this value should be continuous with the rest of the field. The unknown boundary data are extrapolated after having solved system II.

For the G13 moment set, system I can be written as

$$
\begin{gathered}
\frac{\partial \rho^{(n)}}{\partial t}+\frac{\partial \rho^{(n)} v_{i}^{(n)}}{\partial x_{i}}=0, \\
\frac{\partial \rho^{(n)} v_{i}^{(n)}}{\partial t}+\frac{\partial\left(\rho^{(n)} v_{i}^{(n)} v_{j}^{(n)}+\sigma_{i j}^{(n)}\right)}{\partial x_{j}}=0, \\
\frac{3 R}{2}\left(\frac{\partial \rho^{(n)} T^{(n)}}{\partial t}+\frac{\partial \rho^{(n)} v_{j}^{(n)} T^{(n)}}{\partial x_{j}}\right)+\sigma_{i j}^{(n)} \frac{\partial v_{i}^{(n)}}{\partial x_{j}}+\frac{\partial q_{j}^{(n)}}{\partial x_{j}}=0, \\
\tau_{i j}^{(n)}=-\tau_{\mathrm{c}}\left(\frac{\partial \tau_{i j}^{(n-1)}}{\partial t}+\frac{\partial \tau_{i j}^{(n-1)} v_{k}^{(n)}}{\partial x_{k}}+\frac{4}{5} \frac{\partial q_{<i}^{(n-1)}}{\partial x_{j>}}+2 p^{(n)} \frac{\partial v_{<i}^{(n-1)}}{\partial x_{j>}}+2 \tau_{k<i}^{(n-1)} \frac{\partial v_{j>}^{(n)}}{\partial x_{k}}\right)
\end{gathered}
$$


and

$$
\begin{aligned}
q_{i}^{(n)}= & -\frac{\tau_{\mathrm{c}}}{\operatorname{Pr}}\left(\frac{\partial q_{i}^{(n-1)}}{\partial t}+\frac{\partial q_{i}^{(n-1)} v_{k}^{(n)}}{\partial x_{k}}+\frac{1}{2} \frac{\partial \rho_{r r<i k>}^{(n-1)}}{\partial x_{k}}+\frac{1}{6} \frac{\partial \rho_{r r s s}^{(n)}}{\partial x_{i}}-\frac{5}{2} \frac{p^{(n)}}{\rho^{(n)}} \frac{\partial p^{(n)}}{\partial x_{i}}-\frac{5}{2} \frac{p^{(n)}}{\rho^{(n)}} \frac{\partial \tau_{i k}^{(n-1)}}{\partial x_{k}}\right. \\
& \left.-\frac{\tau_{i k}^{(n-1)}}{\rho^{(n)}} \frac{\partial p^{(n)}}{\partial x_{k}}-\frac{\tau_{i j}^{(n-1)}}{\rho^{(n)}} \frac{\partial \tau_{j k}^{(n-1)}}{\partial x_{k}}+\frac{7}{5} q_{k}^{(n-1)} \frac{\partial v_{i}^{(n)}}{\partial x_{k}}+\frac{2}{5} q_{k}^{(n-1)} \frac{\partial v_{k}^{(n)}}{\partial x_{i}}+\frac{2}{5} q_{i}^{(n-1)} \frac{\partial v_{k}^{(n)}}{\partial x_{k}}\right),
\end{aligned}
$$

whereas system II can be expressed as follows:

$$
\tau_{i j}^{(n)}=-\tau_{c} w_{n}\left(\frac{\partial \tau_{i j}^{(n)}}{\partial t}+\frac{\partial \tau_{i j}^{(n)} v_{k}^{(n)}}{\partial x_{k}}+\frac{4}{5} \frac{\partial q_{<i}^{(n)}}{\partial x_{j>}}+2 p^{(n)} \frac{\partial v_{<i}^{(n)}}{\partial x_{j>}}+2 \tau_{k<i}^{(n)} \frac{\partial v_{j>}^{(n)}}{\partial x_{k}}\right)+\left(1-w_{n}\right) \tau_{i j}^{(n-1)}
$$

and

$$
\begin{aligned}
q_{i}^{(n)} & =-\frac{\tau_{\mathrm{c}} w_{n}}{\operatorname{Pr}}\left(\frac{\partial q_{i}^{(n)}}{\partial t}+\frac{\partial q_{i}^{(n)} v_{k}^{(n)}}{\partial x_{k}}+\frac{1}{2} \frac{\partial \rho_{r r<i k>}^{(n)}}{\partial x_{k}}+\frac{1}{6} \frac{\partial \rho_{r r s s}^{(n)}}{\partial x_{i}}-\frac{5}{2} \frac{p^{(n)}}{\rho^{(n)}} \frac{\partial p^{(n)}}{\partial x_{i}}-\frac{5}{2} \frac{p^{(n)}}{\rho^{(n)}} \frac{\partial \tau_{i k}^{(n)}}{\partial x_{k}}\right. \\
& \left.-\frac{\tau_{i k}^{(n)}}{\rho^{(n)}} \frac{\partial p^{(n)}}{\partial x_{k}}-\frac{\tau_{i j}^{(n)}}{\rho^{(n)}} \frac{\partial \tau_{j k}^{(n)}}{\partial x_{k}}+\frac{7}{5} q_{k}^{(n)} \frac{\partial v_{i}^{(n)}}{\partial x_{k}}+\frac{2}{5} q_{k}^{(n)} \frac{\partial v_{k}^{(n)}}{\partial x_{i}}+\frac{2}{5} q_{i}^{(n)} \frac{\partial v_{k}^{(n)}}{\partial x_{k}}\right)+\left(1-w_{n}\right) q_{i}^{(n-1)},
\end{aligned}
$$

where the superscript $n$ indicates the iteration index and $w_{n}$ is a monotonically decreasing weight, such that $w_{n}=1 / n$. One whole iteration comprises a complete solution of both sub-systems.

The iterative procedure is initiated at $n=1$ by solving for equilibrium - that is, by setting all non-equilibrium variables in system I to zero, i.e., $\tau_{i j}^{(0)}=0$ and $q_{j}^{(0)}=0$. At $n=1$, it can be shown that system I is equivalent to the NSF equations. Having solved system I, we substitute all variables into system II containing the non-equilibrium components, which are assumed to be continuous up to the boundary. The solution procedure switches between the two sub-systems until the $L_{2}$ error norm, defined for the uncontrollable boundary data in system II over successive iterations, is considered to be sufficiently small [12].

The mathematical details of this iterative procedure have been presented in [10-12]. However, it should be noted that their solutions do not take into account the velocity slip or temperature jump boundary conditions. In this paper, the same method has been used with the incorporation of velocity slip and temperature jump into the solution, together with other modifications that better represent the dynamics of non-equilibrium gas flows.

\section{Phenomenological variants of simple continuum models}

In continuum models, phenomenological techniques are often used to capture a particular physical behavior rather than modelling it from first principles. Such an approach can be adopted to model features in non-equilibrium micro-scale gas flows. Velocity slip, temperature jump, and strain-rate scaling through the Knudsen layer are phenomenological models that will be briefly described here.

\subsection{Velocity slip and temperature jump boundary conditions}

The velocity slip and temperature jump boundary conditions are simplified phenomenological approaches to represent both non-equilibrium and gas-surface interaction effects occurring near solid walls. Such boundary conditions were first suggested by [15] and [19], respectively. Using Grad's closure approximation for the PDF, we find that the boundary conditions accounting for velocity slip and temperature jump are of the form [18]:

$$
\begin{gathered}
p_{\alpha}=p+\frac{1}{2} \tau_{j k} n_{j} n_{k}, \\
\left(v_{i}\right)_{\text {slip }}=\left(v_{i}\right)_{\text {gas }}-\left(v_{i}\right)_{\text {wall }}=-\frac{1}{5} \frac{\left(\alpha_{2} q_{i}-n_{i} q_{k} n_{k}\right)}{p_{\alpha}} \pm \frac{2-\sigma}{\sigma}\left(\frac{\pi R T}{2}\right)^{\frac{1}{2}} \frac{\left(\alpha_{1} \tau_{i j} n_{j}-n_{i} \tau_{j k} n_{j} n_{k}\right)}{p_{\alpha}}
\end{gathered}
$$


and

$$
\frac{T_{\mathrm{wall}}}{T_{\mathrm{gas}}}-1= \pm \frac{2-\sigma_{T}}{\sigma_{T}}\left(\frac{\pi}{2 R T}\right)^{\frac{1}{2}} \frac{1}{2 p_{\alpha}} \beta_{1} q_{k} n_{k}+\frac{1}{4 p_{\alpha}} \tau_{j k} n_{j} n_{k}-\frac{\left(v_{i}\right)_{\text {slip }}\left(v_{i}\right)_{\text {slip }}}{4 R T},
$$

respectively. The subscript 'wall' indicates wall boundary conditions.

Both boundary conditions are extensively used in the slip-flow regime, i.e., $0.001<K n<0.1$ [5] in combination with the NSF equations. Various modifications are also presented in the literature in order to extend their validity into the early transition regime, i.e., $0.1<K n<1.0$ [1]. Nevertheless, modifying such boundary conditions is not sufficient to fully capture many non-equilibrium effects in the transition regime. Further modifications to the governing or constitutive equations are required in order to capture effects such as the non-linear stress/strain-rate and the non-linear heat-flux/temperature-gradient relationships in the proximity of solid walls.

\subsection{The Knudsen layer and constitutive scaling}

Much of the drive behind the extension of slip and jump boundary conditions has been to include the effects of the Knudsen layer - the local region of non-equilibrium flow extending a few molecular mean free paths from solid walls. Modifying the coefficients $\alpha_{1}, \alpha_{2}$ and $\beta_{1}$ in the velocity slip and temperature jump conditions of Eqs. (12) and (13) only changes the magnitude of the wall boundary values. While this may provide a better solution in the bulk flow, it fails to model the effects of the Knudsen layer in the vicinity of the wall.

One possible solution that has been proposed to represent this non-equilibrium region is to introduce a scaling function, $\psi$, in the viscous stress/strain-rate relationship which is dependent on the distance, $\bar{x}_{j}$, from a solid boundary. Any such scaling function should have a large effect within a few mean free paths from the wall and scale to unity in the bulk flow. Cercignani [3] solved the linearized Boltzmann equation and showed that the one-dimensional velocity profile through the Knudsen layer was of the form:

$$
v_{i}\left(\bar{x}_{j}\right)=-\left(\frac{\tau_{i j}}{\mu}\right)\left[\bar{x}_{j}+\xi-\lambda I\left(\frac{\bar{x}_{j}}{\lambda}\right)\right],
$$

where $\xi$ is a constant and $I\left(\bar{x}_{j} / \lambda\right)$ is a correction function for the Knudsen layer. Lockerby et al. [13] identified one such correction function for the momentum Knudsen layer:

$$
I\left(\frac{\bar{x}_{j}}{\lambda}\right) \approx \frac{7}{20}\left(1+\frac{\bar{x}_{j}}{\lambda}\right)^{-2} .
$$

For an essentially one-dimensional flow, the stress tensor then has the scaled form:

$$
\tau_{12}=-\frac{\mu}{\psi} \frac{\mathrm{d} v_{1}}{\mathrm{~d} x_{2}}=-\left(1+\frac{7}{10}\left(1+\frac{\bar{x}_{2}}{\lambda}\right)^{-3}\right)^{-1} \mu \frac{\mathrm{d} v_{1}}{\mathrm{~d} x_{2}}
$$

This representation is similar to other scaling methods used in micro-liquid transport models to describe non-linearities close to the wall [16].

\section{Capturing non-equilibrium effects: a combined technique}

Restricting the modelling of rarefied gas flows to the conservation equations and modifications thereof might hamper the possibility of capturing the correct physics occurring in the transition regime. The combination of conservation equations and scaling methods alone is not enough to capture all non-equilibrium flow effects. Nevertheless, this method can capture some of the stress/strain rate non-linearities occurring in the proximity of solid boundaries.

We, therefore, propose a solution of the moment equations in conjunction with constitutive scaling methods within the Knudsen layer. In a similar manner to the approach described in [13], we introduce a viscosity scaling function in the G13 constitutive relations in order to model non-linearities in the flow close to surfaces. To retain the definition of the Prandtl number for a monatomic gas, $P r=2 / 3$, we shall assume that the thickness of the momentum and thermal Knudsen layers are equivalent. Hence the same scaling function will 


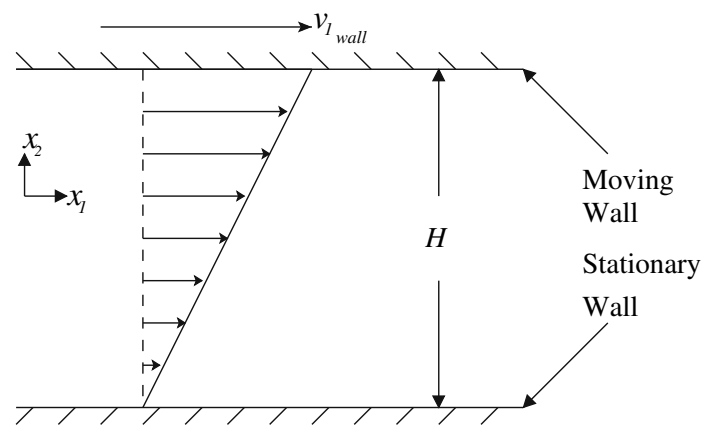

Fig. 1 Schematic representation of Couette flow

be assumed for the momentum flux and thermal heat flux. In reality, this is unlikely since the relaxation times for momentum and thermal energy are different [20].

The viscous stress and heat flux NSF constitutive relations for viscous stress and heat flow have been modified to accommodate the scaling parameter, $\psi$, as follows:

$$
\tau_{i j}=-\frac{2 \mu}{\psi} \frac{\partial v_{<i}}{\partial x_{j>}} \quad \text { and } \quad q_{j}=-\frac{5}{2 P r} \frac{\mu R}{\psi} \frac{\partial T}{\partial x_{j}} .
$$

Similarly, the scaled G13 relations can be writen as

$$
\tau_{i j}=-\frac{\mu}{\psi p}\left(\frac{\partial \tau_{i j}}{\partial t}+v_{k} \frac{\partial \tau_{i j}}{\partial x_{k}}+\frac{4}{5} \frac{\partial q_{<i}}{\partial x_{j>}}+2 p \frac{\partial v_{<i}}{\partial x_{j>}}+\tau_{i j} \frac{\partial v_{k}}{\partial x_{k}}+2 \tau_{k<i} \frac{\partial v_{j>}}{\partial x_{k}}\right)
$$

and

$$
\begin{aligned}
q_{i}= & -\frac{\mu}{\psi p P r}\left(\frac{\partial q_{i}}{\partial t}+v_{k} \frac{\partial q_{i}}{\partial x_{k}}+R T \frac{\partial \tau_{i k}}{\partial x_{k}}-\frac{\tau_{i j}}{\rho} \frac{\partial \tau_{j k}}{\partial x_{k}}+\frac{2}{5} q_{k} \frac{\partial v_{k}}{\partial x_{i}}+\frac{5 R p}{2} \frac{\partial T}{\partial x_{i}}+\frac{7}{5} q_{k} \frac{\partial v_{i}}{\partial x_{k}}+\frac{7}{5} q_{i} \frac{\partial v_{k}}{\partial x_{k}}\right. \\
& \left.+\frac{7 R \tau_{i j}}{2} \frac{\partial T}{\partial x_{k}}-\frac{\tau_{i k}}{\rho} \frac{\partial p}{\partial x_{k}}\right) .
\end{aligned}
$$

\section{Steady Couette flow}

A steady-state Couette flow of argon gas was used as a test case to investigate the various models and the proposed new solution technique. Our results were compared to DSMC data for $K n=0.1$ and $K n=0.5$. The steady-state Couette flow problem is a simplified one-dimensional problem in which $\partial / \partial t, \partial / \partial x_{1}, \partial / \partial x_{3}$, $n_{1}, n_{3}, v_{2}$, and $v_{3}$ are all zero (see Fig. 1). The conservation equations need to be solved for $\rho, v_{1}, T$ and $p$, together with the equation of state for an ideal gas and the constitutive equations for $\tau_{i j}$ and $q_{i}$ that provide a closed set of equations.

For this one-dimensional problem, the conservation equations reduce to

$$
\frac{\partial \tau_{12}}{\partial x_{2}}=0, \quad \frac{\partial p}{\partial x_{2}}+\frac{\partial \tau_{22}}{\partial x_{2}}=0, \quad \frac{\partial \tau_{32}}{\partial x_{2}}=0, \quad \tau_{12} \frac{\partial v_{1}}{\partial x_{2}}-\frac{\partial q_{2}}{\partial x_{2}}=0
$$

and

$$
p=\rho R T .
$$

The velocity slip and temperature jump boundary conditions reduce to

$$
\left(v_{1}\right)_{\mathrm{slip}}=\left(v_{1}\right)_{\mathrm{gas}}-\left(v_{1}\right)_{\mathrm{wall}}= \pm \frac{2-\sigma}{\sigma}\left(\frac{\pi R T}{2}\right)^{\frac{1}{2}} \frac{2 \alpha_{1} \tau_{12}}{2 p+\tau_{22}}-\frac{2 \alpha_{2} q_{1}}{10 p+5 \tau_{22}},
$$

and

$$
\frac{T_{\mathrm{wall}}}{T_{\mathrm{gas}}}-1=\frac{2-\sigma_{T}}{\sigma_{T}}\left(\frac{\pi}{2 R T}\right)^{\frac{1}{2}} \frac{2 \beta_{1} q_{2}+\tau_{22}}{4 p+2 \tau_{22}}-\frac{\left(v_{1}\right)_{\mathrm{slip}}\left(v_{1}\right)_{\mathrm{slip}}}{4 R T} .
$$


If the effects of the Knudsen layer are taken into account using the classical NSF equations, the correction coefficients are $\alpha_{1}=1.114, \alpha_{2}=1.34533$ and $\beta_{1}=1.127$ [18].

The one-dimensional NSF constitutive relations reduce to

$$
\tau_{12}=-\frac{\mu}{\psi} \frac{\partial v_{1}}{\partial x_{2}}, \quad \tau_{22}=0, \quad \tau_{32}=0
$$

and

$$
q_{2}=-\frac{5}{2 \psi} \frac{\mu R}{\operatorname{Pr}} \frac{\partial T}{\partial x_{2}}
$$

whereas the one-dimensional G13 expressions together with Eq. (20) reduce to

$$
\begin{aligned}
\tau_{12} & =-\frac{\mu}{\psi p}\left(\frac{2}{5} \frac{\partial q_{1}}{\partial x_{2}}+\left(\tau_{22}+p\right) \frac{\partial v_{1}}{\partial x_{2}}\right), \\
\tau_{22} & =\frac{\mu \tau_{12}}{\psi p}\left(\frac{6}{5} \frac{\partial v_{1}}{\partial x_{2}}\right), \quad \tau_{11}=-\frac{4}{3} \tau_{22}, \\
q_{1} & =-\frac{\mu}{\psi p P r}\left(\frac{7 q_{2}}{5} \frac{\partial v_{1}}{\partial x_{2}}+\frac{7 R \tau_{12}}{2}\left(\frac{\partial T}{\partial x_{2}}\right)\right)
\end{aligned}
$$

and

$$
q_{2}=-\frac{\mu}{\psi p \operatorname{Pr}}\left(\frac{2}{5} q_{1} \frac{\partial v_{1}}{\partial x_{2}}+R T \frac{\partial \tau_{22}}{\partial x_{2}}+R\left(\frac{7 \tau_{22}}{2}+\frac{5 p}{2}\right) \frac{\partial T}{\partial x_{2}}\right) .
$$

If there is no scaling of the stress/strain relationship, the function $\psi$ is set to unity.

\section{Results}

The G13 equations and their modifications have been solved using differential iteration, in which system I is the governing and constitutive equation set resolving $\rho, v_{1}, T, p, \tau_{12}$ and $q_{2}$. The production terms of the uncontrollable boundary data, i.e., $\tau_{11}, \tau_{22}$ and $q_{1}$, are computed in system II and weighted values thereof appear as source terms in system I in the next iteration. A finite volume technique was used to solve system I, whereas system II was solved once for every converged solution of system I by a central difference approximation of the derivatives. The whole system of equations converges to a final steady-state solution by reducing the $L_{2}$ error norm of the uncontrollable boundary data, $E r$, between subsequent iterations as defined by [11]:

$$
E r=\frac{\left\|u^{(n)}-u^{(n-1)}\right\|}{w_{n}},
$$

where

$$
\left\|u^{(n)}\right\|=\left(\int_{0}^{1}\left(\Omega_{22}^{(n)^{2}}+Q_{1}^{(n)^{2}}\right) \mathrm{d} X_{2}\right)^{-\frac{1}{2}}
$$

with

$$
\Omega_{i j}=\frac{\tau_{i j}}{P} \quad \text { and } \quad Q_{j}=\frac{q_{j}}{P\left(R T_{\text {wall }}\right)^{\frac{1}{2}}} .
$$

In addition, the problem has been normalized as follows:

$$
X_{i}=\frac{x_{i}}{H}, \quad V_{i}=\frac{v_{i}}{v_{i_{\text {wall }}}}, \quad \Theta=\frac{T}{T_{\text {wall }}},
$$

where $P=p+\tau_{22}$ is the total prescribed normal stress obtained as a constant of integration from Eq. (20). For argon, the specific gas constant, $R=207.83 \mathrm{~J} \mathrm{~K}^{-1} \mathrm{~kg}^{-1}$.

The following boundary conditions were used for all test cases: at $X_{2}=0, V_{1}=0$ and $\Theta=1$ whereas at $X_{2}=1, V_{1}=1$ and $\Theta=1$. Full accommodation was assumed at the walls, i.e., $\sigma=1$ and $\sigma_{T}=1$. Two flow regimes were considered, namely $K n=0.1$ and $K n=0.5$, and the results are presented in Figs. 2 and 3 . 


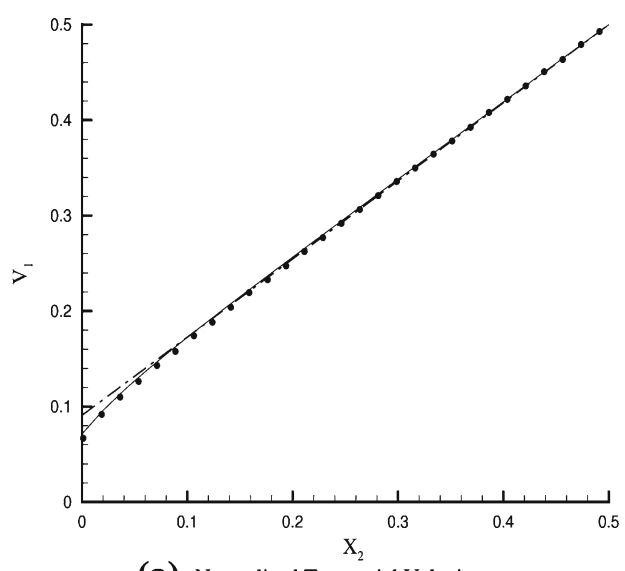

(a) Normalised Tangential Velocity

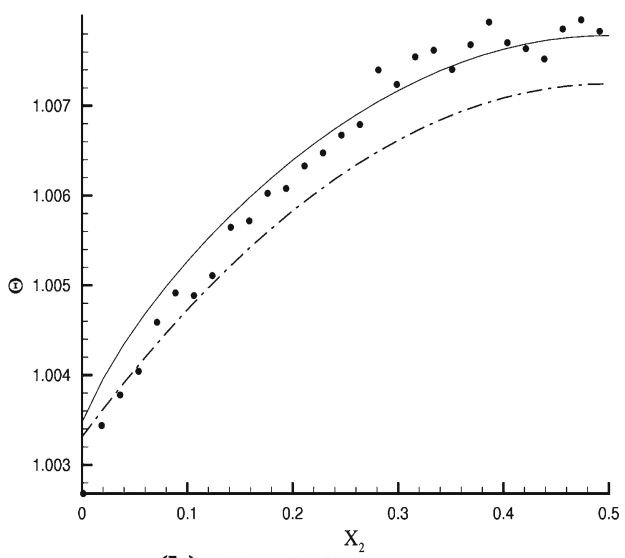

(b) Normalised Temperature

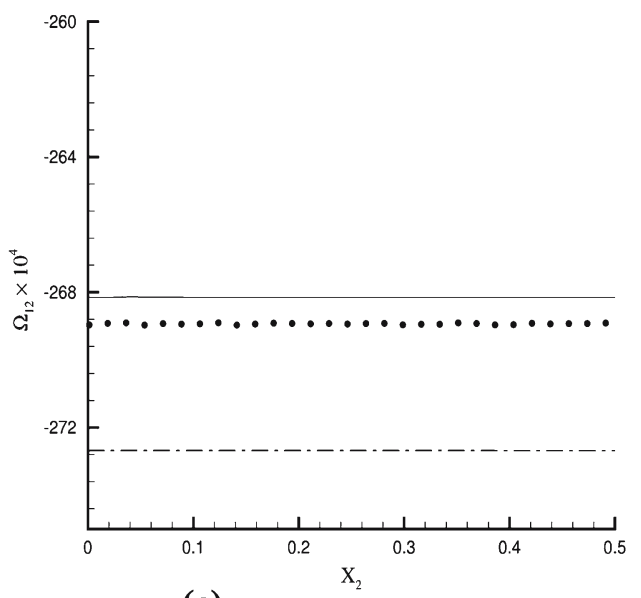

(c) Normalised Shear Stress

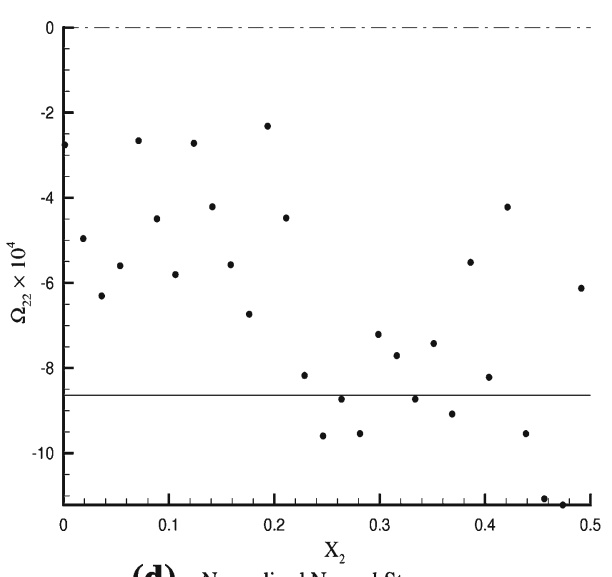

(d) Normalised Normal Stress

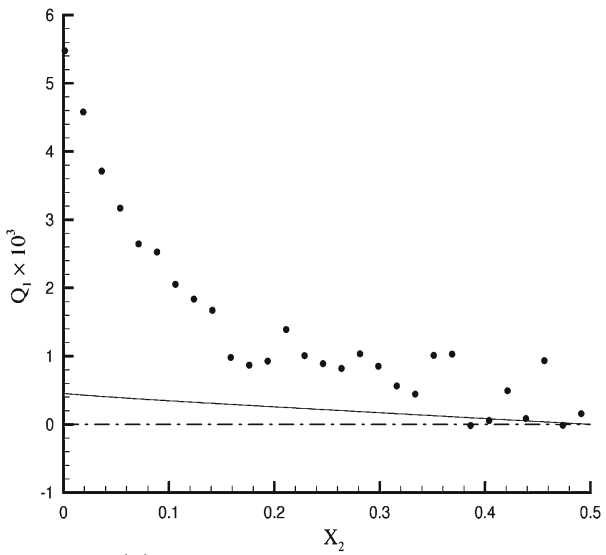

(e) Normalised Tangential Heat Flux

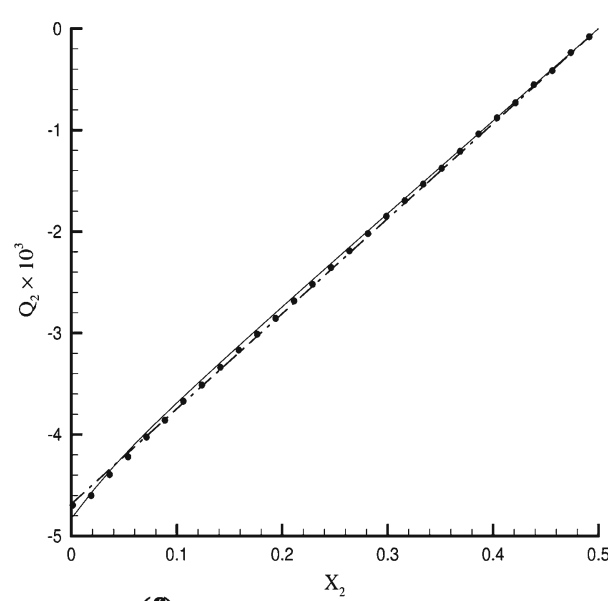

(f) Normalised Normal Heat Flux

Fig. 2 Couette flow results for $K n=0.1$; DSMC predictions ( filled circle), NSF solution with conventional slip boundary conditions (dashed dotted line), and combined G13 and Knudsen layer wall scaling solution (solid line)

In both these cases, the Mach number, defined as $M a=v_{i_{\text {wall }}} / \sqrt{2 R T_{\text {wall }}}$, was approximately 0.3 . The results are shown in half-space for clarity, and compare the DSMC predictions to the NSF solution with conventional slip/jump boundary conditions and the new solution to the G13 equations for both test cases. In Fig. 3, for the $K n=0.5$ case, the conventional G13 solution is additionally shown to highlight the relative differences between the phenomenological scaling and extended constitutive terms. 


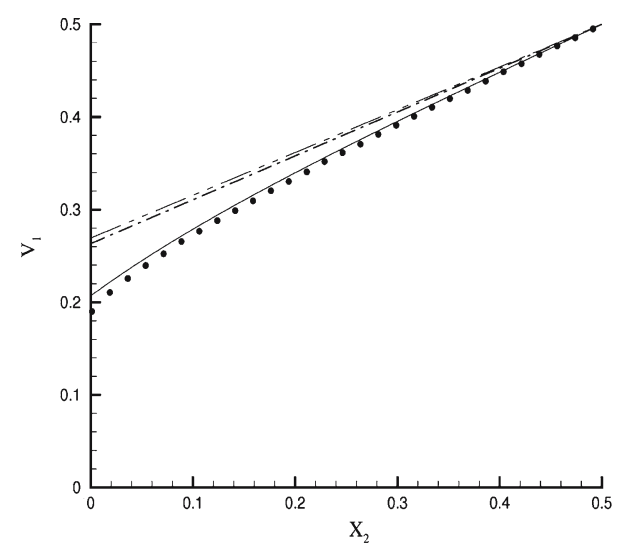

(a) Normalised Tangential Velocity

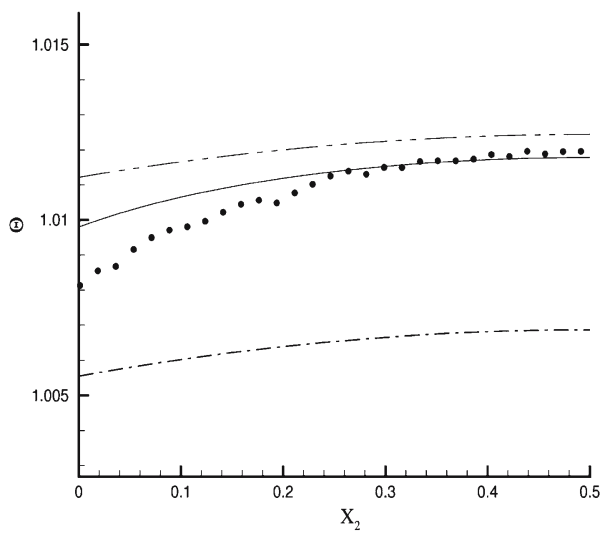

(b) Normalised Temperature

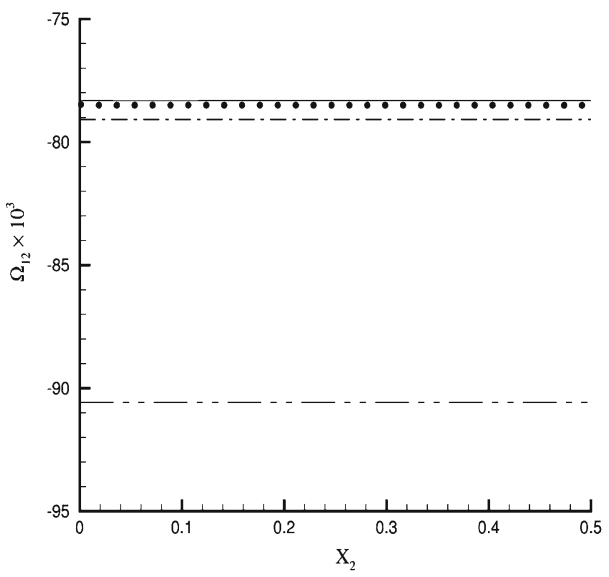

(c) Normalised Shear Stress

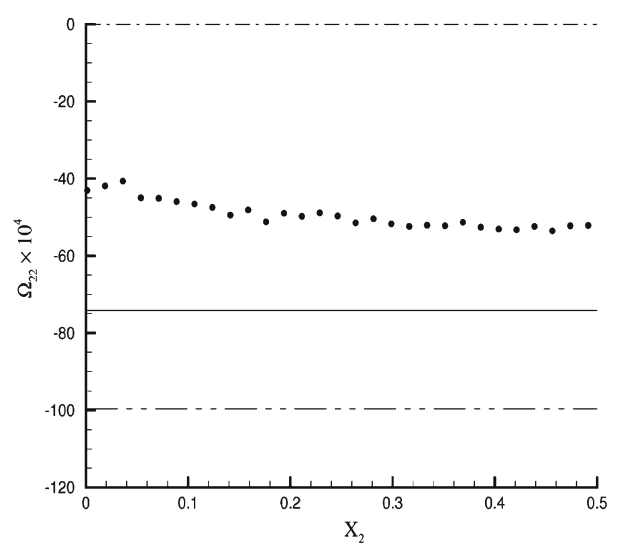

(d) Normalised Normal Stress

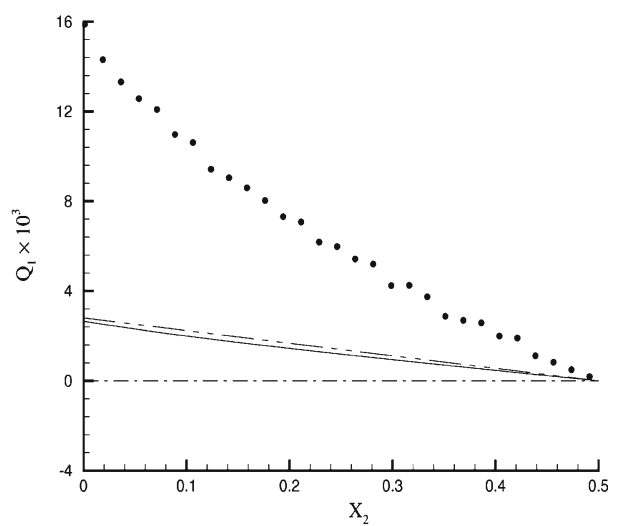

(e) Normalised Tangential Heat Flux

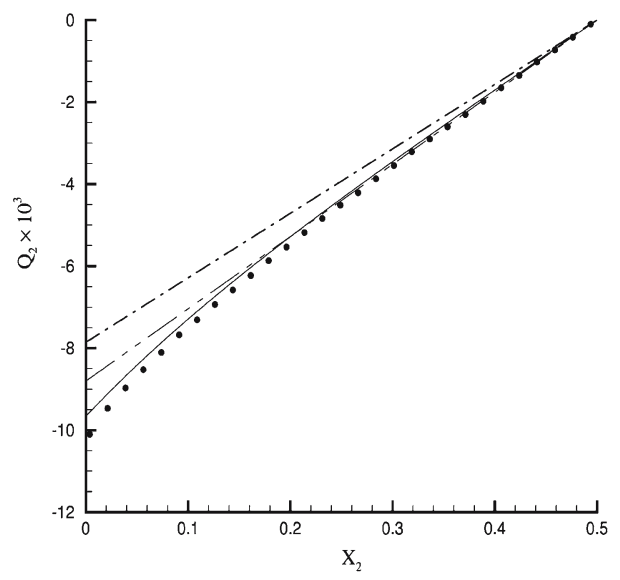

(f) Normalised Normal Heat Flux

Fig. 3 Couette flow results for $K n=0.5$; DSMC predictions ( filled circle), NSF solution with conventional slip boundary conditions (dashed dotted line), G13 solution with conventional slip boundary conditions (dashed line), and combined G13 and Knudsen layer wall scaling solution (solid line)

\section{Discussion and conclusions}

Figures 2 and 3 show that the combination of the G13 equations with scaling functions achieves a better representation of the Knudsen layer in the near wall region than the standard NSF slip-flow solution. In particular, we see that: 
(1) The non-linearity near the wall for the tangential velocity, $V_{1}$, is better predicted (see Figs. $2 \mathrm{a}$ and $3 \mathrm{a}$ ) due to the inclusion of the wall scaling function in the stress constitutive law.

(2) The method achieves a better representation of the temperature profile in the bulk flow due to an improved prediction of the normal heat flux, $Q_{2}$. As shown in Fig. 3f, this is partly due to the extended constitutive terms in the G13 equations and further improved by the wall scaling function.

(3) The proposed method achieves a better estimate of the shear stress, which in turn yields a better estimate of the velocity slip at the wall. This improvement is solely attributed to the constitutive scaling.

(4) The proposed method captures some of the non-equilibrium effects which are otherwise absent from NSF solutions. In particular, the method manages to capture the normal stresses, $\Omega_{11}$ and $\Omega_{22}$. Additionally, the trend for tangential heat flux, $Q_{1}$ is better represented in the proposed method. Such effects can only be captured through the inclusion of 13-moments or more. The combination of the NSF approach with the Knudsen layer wall function technique is unable to capture such non-equilibrium behavior.

These phenomena are not appreciable at low Knudsen numbers and, in particular, Fig. 2d shows a scattered result obtained by DSMC. This is due to the fact that the modelled gas is not very rarefied and the average flow speed is fairly low, typical of applications in MEMS. From the DSMC predictions it can also be seen that the tangential heat flux, $Q_{1}$, is of the same order of magnitude as the normal heat flux, $Q_{2}$, underlining the importance of capturing such effects since they play an implicit role in the conservation equations. Although it is not part of the present investigation, it is likely that the magnitude of quantities such as stress and heat flux will be affected by the Mach number.

The computational expense of the proposed approach is of the same order as a classical NSF solver, making it attractive for engineering design applications in the early transition regime. The computational overhead of using such a technique in comparison to the NSF/slip solution is problem-dependent. In particular, for these specific test cases, it has been found that for $K n=0.1$ and a relative converged error change of $E r=10^{-5}$, the increase in CPU time is approximately $25 \%$ whereas for $K n=0.5$, with the same relative error threshold, the additional CPU overhead is approximately $90 \%$.

This novel technique still has some shortcomings. In particular, it can be shown that the proposed solution method for the G13 equations is incapable of capturing non-linearities of normal stress and tangential heat flux occurring in the vicinity of the wall. Currently, the iteration method is suitable for flows that have a steady-state solution. An extension to time-dependent solutions would incur extra computational cost since the system of equations needs to converge iteratively on both systems for each time step. Two possible ways of improving the current approach are by considering higher-order formulations, such as the regularized 13-moment equations [18] or Grad's 26-moment equations or using alternative wall functions for the different moments in the G13 equations.

Acknowledgments The authors would like to thank X. J. Gu for providing the DSMC data. Additionally, we would like to thank Y. Zheng and L. O'Hare for the informative discussions. This work was funded by the Engineering and Physical Sciences Research Council (EPSRC) under grant no. GR/S77196/01. Additional support was provided by EPSRC under the auspices of CCP12.

\section{References}

1. Barber, R.W., Emerson, D.R.: Challenges in modeling gas-phase flow in microchannels: from slip to transition. Heat Transf. Eng. 27(4), 3-12 (2006)

2. Bird, G.A.: Molecular Gas Dynamics and the Direct Simulation of Gas Flows. Clarendon Press, Oxford (1994)

3. Cercignani, C.: Rarefied Gas Dynamics-From Basic Concepts to Actual Calculations. Cambridge University Press, Cambridge (2000)

4. Chapman, S., Cowling, T.G.: The Mathematical Theory of Non-uniform Gases. Cambridge University Press, Cambridge (1991)

5. Gad-el-Hak, M.: The fluid mechanics of microdevices-The Freeman scholar lecture. ASME J. Fluids Eng. 121, 5-33 (1999)

6. Grad, H: On the kinetic theory of rarefied gases. Commun. Pure Appl. Math. 2, 331-407 (1949)

7. Gu, X.J., Emerson, D.R.: A computational strategy for the regularized 13 moment equations with enhanced wall-boundary conditions. J. Comput. Phys. 225, 263-283 (2007)

8. Ikenberry, E., Truesdell, C.: On the pressures and the flux of energy in a gas according to Maxwell's kinetic theory I \& II. J. Ration. Mech. Anal. 5, 1-128 (1956)

9. Kogan, M.N.: Rarefied Gas Dynamics. Plenum Press, New York (1969)

10. Liu I.-S.: On well-posedness of classical boundary conditions in extended thermodynamics. In: Wang, Y., Hutter, K. (eds.), Proceedings of the XIVth International Symposium on Trends in Applications of Mathematics to Mechanics, pp. 225-233, Shaker Verlag, Aachen, 2005 
11. Liu, I.-S.: A method of differential iteration for boundary value problems in extended thermodynamics. Nonlinear Anal. Real World Appl. 8, 1113-1131 (2007)

12. Liu, I.-S., Rincon, M.A.: A boundary value problem in extended thermodynamics—one-dimensional steady flows with heat conduction. Continuum Mech. Thermodyn. 16, 109-124 (2004)

13. Lockerby, D.A., Reese, J.M., Gallis, M.A.: Capturing the Knudsen layer in continuum-fluid models of non-equilibrium gas flows. AIAA J. 43(6), 1391-1393 (2005)

14. Lockerby, D.A., Reese, J.M., Gallis, M.A.: The usefulness of higher-order constitutive relations for describing the Knudsen layer. Phys. Fluids 17, Art. No. 100609 (2005)

15. Maxwell, J.C.: On stresses in rarefied gases arising from temperature inequalities. Phil. Trans. R. Soc. Ldn 170, 231$256(1879)$

16. Pfahler J.N.: Liquid transport in micron and submicron size channels. Ph.D. Thesis. University of Pennsylvania, PA, USA, 1992

17. Rapaport, D.C.: The Art of Molecular Dynamics Simulation. Cambridge University Press, Cambridge (2004)

18. Struchtrup, H.: Macroscopic Transport Equations for Rarefied Gas Flows. Springer, Germany (2005)

19. von Smoluchowski, M.: Über Wärmeleitung in verdünnten Gasen. Ann. Phys. Chem. 64, 101-130 (1898)

20. Woods, L.C.: An Introduction to the Kinetic Theory of Gases and Magnetoplasmas. Oxford University Press, Oxford (1993) 